\title{
Results of sımultaneous pancreas resectıon after neoad- juvant chemotherapy in local advenced gastric cancers
}

\author{
Murat KALIN ${ }^{1}$, Pirilti ÖZCAN ${ }^{1}$, Özgül DÜZGÜN ${ }^{* 2}{ }^{2}$, Ömer Faruk ÖZKAN ${ }^{1}$ \\ 'Department of General Surgery, Health Sciences University Ümraniye Research and Training Hospital İstanbul, Istanbul, Turkey \\ ${ }^{2}$ Department of Surgical Oncology, Health Sciences University Ümraniye Research and Training Hospital İstanbul, Istanbul, Turkey
}

Introduction: Standard treatment in gastric cancer gained a new dimension after contributions from South Korea and Japanese groups. Advanced stage non metastatic gastric cancer which was accepted as an inoperability criteria before 2000s, can nowadays be accepted as resectable due to advancements in neoadjuvant chemotherapy and aggressive surgical methods. In this study, we aim to analyze the results of simultaneous pancreas resection in patients with T4b tumor with adjacent organ invasion.

Methods: Local advanced gastric cancer cases which were collected prospectively between 2016 to 2020 were analyzed. Among 70 patients with local advanced gastric cancer, 14 of them with simultaneous pancreas invasion were separated and evaluated on the basis of demographic data, postoperative complications, neoadjuvant chemotherapy modalities.

Results: Among 70 patients which were operated between years 2016 to 2020 due to local advanced gastric cancer, 14 of them had pancreas invasion. Average age of these 14 patients was 58 (46 to 72), male/female ratio was: 5/9. Among these, whipple was performed in 4 (28.57\%), subtotal pancreatectomy was performed in 5 (35.71\%) and distal pancreatectomy was performed in 5 (35.71\%) in addition to total gastrectomy and D2 lenf dissection. Splenectomy was performed simultaneously in 8 cases of subtotal and distal pancreatectomy. Mean operation time forall 14 caseswas $317 \pm 33$ minutes. When postoperative complications were analyzed, 2 cases had biliary leakage, 2 cases.

Conclusions: We think that simultaneous resection of pancreas in local advanced gastric cancers can be performed in surgical oncology clinics with contributions of experienced hepatobiliary surgeons with acceptable morbidity and mortality rate. 